\title{
EVALUACIÓN DEL RENDIMIENTO DE UNA RED LAN SOBRE POWER LINE COMMUNICATIONS PARA LA TRANSMISIÓN DE VOIP
}

\author{
EVALUATION OF THE PERFORMANCE OF A NETWORK \\ LAN OVER POWERLINE COMMUNICATIONS FOR THE \\ TRANSMISSION OF VOIP
}

\author{
Juan Carlos Vesga-Ferreira \\ M. Sc. Telecomunicaciones \\ Universidad Nacional Abierta y a Distancia \\ Bucaramanga, Colombia \\ juan.vesga@unad.edu.co
}

\author{
Gerardo Granados-Acuña \\ M. Sc. Telemática \\ Universidad Nacional Abierta y a Distancia \\ Bucaramanga, Colombia \\ gerardo.granados@unad.edu.co
}

\author{
José Antonio Vesga-Barrera \\ M. Sc. Potencia Eléctrica \\ Corporación Universitaria de Ciencia y Desarrollo \\ Bucaramanga, Colombia \\ jvesgabarrera@yahoo.es
}

\begin{abstract}
Resumen- La calidad de servicio (QOS) se define como la capacidad que tiene una red para administrar la demanda de tráfico según la clase de servicio, con el fin de satisfacer las expectativas del usuario y de acuerdo con unas métricas claramente establecidas, lo que aplica inclusive para las redes con tecnología PLC. Ante la necesidad de optimizar QoS en redes IP se plantea un diseño experimental de tipo factorial mixto, con el fin de evaluar el rendimiento de una red PLC para la transmisión de voz sobre IP, en términos del throughput, latencia y jitter, en un entorno residencial y con el uso de adaptadores PLC soportados en el estándar HomePlug AV. EI resultado del experimento no solo establece el grado de influencia desde el punto de vista estadístico que puede presentar el tipo de codec utilizado o el número de estaciones activas, sino que permite identificar cuáles son los codecs más recomendados para transmitir voz sobre IP, mejorando el rendimiento de la red y garantizando adecuados niveles de QoS.
\end{abstract}

Palabras clave- ANOVA, calidad de servicio, comunicaciones por línea de potencia, diseño experimental, HomePlug AV, rendimiento de una red.

Abstract-The quality of service (Q०S) is defined as the ability of a network to manage the traffic demand according to the class of service, in order to meet the expectations of the user and in accordance with a clearly established metrics, which applies even for networks with PLC technology. Faced with the need to optimize QoS in IP networks, a experimental design type mixed factorial is presented to evaluate the performance of a PLC network to the Voice over IP transmission, in terms of throughput, latency and jitter, in a residential environment and with the use of PLC adapters supported in the HomePlug AV standard. The result of the experiment not only establishes the degree of influence from the statistical point of view that you can produce the type of codec used or the number of active stations, but that allows you to identify which are the most recommended codecs to transmit voice over IP, improving the performance of the network and ensuring adequate levels of QoS.

Keywords- ANOVA, experimental design, HomePlug AV, powerline communications, network performance, QoS.

\section{INTRODUCCIÓN}

La red inteligente (Smart Grid) corresponde a un nuevo concepto de la red eléctrica, donde convergen la prestación de servicios energéticos y de comunicaciones, soportados en IP y unificados entre sí como una red integral, la cual tiene como principal objetivo mejorar la eficiencia y la fiabilidad de la red eléctrica, adaptándola a las necesidades de la era digital [1]. Uno de los aspectos de mayor discusión en el interior de Smart Grid ha sido la selección de la tecnología por emplear 
en el sistema de comunicación, donde el uso de tecnologías inalámbricas y PLC (Power Line Communications) presentan actualmente una mayor aceptación, debido a las prestaciones que estas tecnologías ofrecen en relación con los procesos de instalación, movilidad, ubicuidad y estabilidad [2].

Power Line Communications hace referencia a un grupo de tecnologías que permiten establecer procesos de comunicación mediante el uso de la red eléctrica como medio físico de transmisión, en donde HomePlug AV (HPAV) es uno de los estándares de mayor importancia en la tecnología PLC, el cual emplea OFDM (Orthogonal Frequency Division Multiplexing) como técnica de multiplexación de la información sobre el canal PLC, opera en el rango de $1.8 \mathrm{MHz}$ a $28 \mathrm{MHz}$, utiliza CSMA/ CA y TDMA como mecanismo de acceso al medio, donde CSMA/CA está destinado para la transmisión de paquetes de datos, mientras que TDMA se utiliza para la transmisión de paquetes de voz $y$ video, con el fin de ofrecer adecuados niveles de QoS [3]. Un aspecto importante es que HPAV no cuenta con un mecanismo de optimización de recursos adecuado para un entorno multiusuario, lo cual afecta considerablemente el rendimiento de la red a medida que aumenta el número de usuarios, debido a que solo uno de los nodos puede transmitir a la vez [4].

Uno de los aspectos de mayor relevancia es el comportamiento de la tecnología PLC para la transmisión de información, considerando el hecho de que la eficiencia de la red depende de las condiciones en que se encuentre la red eléctrica [5], [6], teniendo en cuenta que la red eléctrica es considerada un medio hostil para la transmisión de información en la que se pueden presentar numerosos problemas en el momento de establecer un proceso de comunicación, como: alto componente de ruido, impedancia variable y posibles atenuaciones significativas [7] En Colombia y en muchos países, el cableado eléctrico no ha sido modificado, ni adaptado para el uso eficiente de esta tecnología, como ha ocurrido en países como Alemania, donde se han realizado diversos estudios y ajustes a su infraestructura en las redes de potencia con el fin de optimizar su comportamiento y eficiencia de transmisión, garantizando niveles de confiabilidad y seguridad [8], [9].
La mayoría de planes del gobierno nacional y departamental de Colombia contemplan el establecimiento y fortalecimiento de políticas públicas encaminadas a la difusión de la conectividad. Uno de los programas relevantes es el Plan Nacional de TIC en el que se busca fortalecer los procesos de conectividad en las organizaciones y favorecer las oportunidades de acceso a tecnologías de punta como PLC [10]. Por otro lado, el plan "Vive Digital", en su numeral 8.1.5 destaca la importancia de establecer estándares de conectividad para entornos residenciales y empresariales "Actualmente, la prestación de servicios de telecomunicaciones para hogares y MiPyME se encuentra limitada por las dificultades para desplegar infraestructura de conectividad al interior de construcciones residenciales y comerciales. Hace falta estandarización y normatividad que facilite el despliegue de infraestructura al interior de las viviendas y edificaciones" [11]. Bajo la premisa anterior, PLC se convierte en una importante alternativa para cumplir con el objetivo mencionado. Adicionalmente, la tecnología PLC puede ser muy apropiada para alcanzar la convergencia en redes de área local como se establece en el documento Visión Colombia 2019 "El sector de las telecomunicaciones deberá contribuir a consolidar una sociedad informada, conectada e integrada al entorno global, para lo cual deberá incorporar continuamente los últimos desarrollos tecnológicos, particularmente adecuarse a la convergencia de redes, terminales y servicios" [12].

Con base en los argumentos precedentes, la implementación de una tecnología como PLC en un país en vías de desarrollo como Colombia se convierte en un reto $\mathrm{y}$, a la vez en una importante alternativa para la expansión de redes LAN en hogares y empresas donde se desee evitar cableado adicional, reducir costos y obtener los beneficios de banda ancha. Ante esta situación, es necesario desarrollar estudios que permitan establecer la viabilidad de la tecnología PLC en el país, tomando como base la implementación de estándares de aceptación y reconocimiento a nivel mundial como HomePlug AV. Por otro lado, las aplicaciones multimedia, audio y voz se convierten cada día en un tema de mayor investigación e interés en el contexto de PLC, debido a los requerimientos de calidad de servicio que la red deberá garantizar, como: capacidad del canal, latencia y jitter; para 
un óptimo desempeño [13]. En vista de lo anterior, surge la pregunta ¿será que una red PLC en entornos residenciales podrá ofrecer adecuados niveles de QoS para la transmisión de voz sobre IP?

De acuerdo con los argumentos expuestos antículo consiste en evaluar el rendimiento de una red LAN en un entorno residencial, operando bajo el estándar HomePlug AV, para la transmisión de voz sobre el protocolo IP. Los resultados de este trabajo pueden ser considerados como relevantes dentro de la tecnología PLC, teniendo en cuenta que aunque mundialmente existe un buen acervo documental relacionado con la aplicación del estándar HomePlug AV, no se encuentran estudios específicos sobre el rendimiento del estándar con respecto al tráfico de VoIP, ni su impacto en redes de área local tanto residenciales como empresariales.

\section{CONTENIDO}

\subsection{Voz sobre IP}

En el website de la Comisión Federal de Comunicaciones de Estados Unidos (FCC / Federal Commission Communications), se define Voz sobre IP (VoIP) como una tecnología que permite el enrutamiento de comunicaciones de voz sobre Internet o a través de una red de computadores [14]. El encargado del transporte de las llamadas en una red que utiliza VolP es el protocolo de internet (IP), perteneciente a la pila de protocolos TCP/ teriormente, el objetivo principal del presente ar-

IP (Transmision Control Protocol / Internet Protocolo). IP provee un conjunto de facilidades para administrar el envío de información de voz sobre redes de datos, de tal manera que la voz proveniente de una llamada es digitalizada y segmentada en paquetes, los cuales viajan por múltiples rutas en las redes de computadores y se reensamblan en el equipo destino, dando la sensación de cero interrupción.

La voz humana (analógica) para ser transmitida eficientemente en redes de datos necesita una transformación a formato digital, mediante un proceso reconocido como digitalización de voz y el cual es realizado por un dispositivo denominado CoDec (Codificador / Decodificador). Los codec pueden ser caracterizados de acuerdo con los siguientes factores:

- La tecnología (de "forma de onda", de "síntesis de voz")

- La tasa de bits: usualmente expresada en bits por segundo (bps)

- La calidad resultante del audio codificado

- La complejidad

- El retardo que introducen: expresado en milisegundos (ms)

De acuerdo con el ancho de banda de la señal de entrada, los codec más representativos del mercado se clasifican en codec de banda angosta y codec de banda ancha. En las Tablas I y II se presentan los codec más representativos para cada caso

TABLA I

CODECS DE BANDA ANGOSTA

\begin{tabular}{|c|l|c|c|l|}
\hline Codec & \multicolumn{1}{|c|}{ Nombre } & Tasa de Bits (kbs) & $\begin{array}{c}\text { Latencia } \\
(\mathrm{ms})\end{array}$ & Observaciones \\
\hline G.711 & PCM: Pulse Code Modulation & 64,56 & 0.125 & $\begin{array}{l}\text { Codec “base”, utiliza dos posibles leyes de compre- } \\
\text { sión: } \mu \text {-law y A-law }\end{array}$ \\
\hline G.723.1 & Hybrid MPC-MLQ and ACELP & $6.3,5.3$ & 37.5 & $\begin{array}{l}\text { Desarrollado originalmente para videoconferencias } \\
\text { en la PSTN, es altamente usado en sistemas VolP }\end{array}$ \\
\hline G.728 & $\begin{array}{l}\text { LD-CELP: Low-Delay code } \\
\text { excited linear prediction }\end{array}$ & $40,16,12.8,9.6$ & 1.25 & $\begin{array}{l}\text { Creado para aplicaciones DCME (Digital Circuit } \\
\text { Multiplex Encodig) }\end{array}$ \\
\hline G.729 & $\begin{array}{l}\text { CS-ACELP: Conjugate Structure } \\
\text { Algebraic Codebook Excited } \\
\text { Linear Prediction }\end{array}$ & $11.8,8,6.4$ & 15 & $\begin{array}{l}\text { Ampliamente utilizado en aplicaciones de VolP a 8 } \\
\text { kbps }\end{array}$ \\
\hline AMR & Adaptive Multi Rate & 12.2 a 4.75 & 20 & Utilizado en redes celulares GSM \\
\hline
\end{tabular}

Fuente: autores. 
Dentro de la categoría de banda angosta se incluyen los codec cuya frecuencia de muestreo de voz es a 8000 muestras por segundo. Para el caso de banda ancha, las frecuencias son bastante mayores y oscilan entre 16000 y 32000 muestras por segundo.

TABLA II

CODECS DE BANDA ANCHA

\begin{tabular}{|c|l|c|c|l|}
\hline Codec & \multicolumn{1}{|c|}{ Nombre } & Tasa de Bits (kbps) & $\begin{array}{c}\text { Latencia } \\
(\mathrm{ms})\end{array}$ & \multicolumn{1}{c|}{ Observaciones } \\
\hline G.722.1 & Transform Coder & 24,32 & 40 & Útil en audio y videoconferencias \\
\hline G.722.2 & AMR-WB & 6.6 a 23.85 & 25.9375 & $\begin{array}{l}\text { Estándar en común con 3GPP y con gran inmunidad } \\
\text { los ruidos de fondo especialmente en ambientes } \\
\text { adversos (ej: celulares) }\end{array}$ \\
\hline G.711.1 & Wideband G.711 & $64,80,96$ & 11.875 & $\begin{array}{l}\text { Amplia el ancho de banda del Codec 711, optimi- } \\
\text { zando su uso para VolP }\end{array}$ \\
\hline G.729.1 & Wideband G.729 & 8 a 32 & $<49$ & $\begin{array}{l}\text { Amplia el ancho de banda del Codec G.729, optimi- } \\
\text { zando su uso para VolP }\end{array}$ \\
\hline RtAudio & Real Time Audio & $8.8,18$ & 40 & $\begin{array}{l}\text { Codec propietario de Microsoft, usado en comunica- } \\
\text { ciones unificadas (OCS) }\end{array}$ \\
\hline
\end{tabular}

Fuente: autores.

Con los codec de banda ancha se incrementa la calidad del sonido, y por ello se genera una gran cantidad de datos, los cuales a su vez requieren un mayor ancho de banda para su transporte y esto hace que sean adecuados solo para sistemas VoIP en redes LAN y WAN de alta velocidad [15]. De acuerdo con su adecuación y utilización para VoIP, los codecs usualmente más relacionados con este tipo de tecnología son, respectivamente G711 [16], G723 [17] y G729 [18]. Por otro lado, el proceso de digitalización de la voz comprende tres etapas: muestreo, cuantificación y codificación:

- Muestreo: Se toman muestras de la señal de voz a intervalos periódicos. Se recomienda que los intervalos estén en coherencia con el teorema del muestreo, el cual indica que la frecuencia mínima a la que puede ser muestreada una señal para luego ser reconstruida, debe ser el doble de la frecuencia máxima de dicha señal [19].

- Cuantificación: Para cada valor muestreado en la etapa anterior se realiza una cuantificación en unidades discretas. La cantidad total de valores discretos debe minimizar el posible ruido de cuantificación.

- Codificación: Los valores cuantificados se codifican en números que pueden ser posteriormente transmitidos y procesados digitalmente. Esta función es en particular realizada por el codec de voz utilizado.

\subsection{Capacidad de canal requerido para transmisión de Voz en IP}

Teniendo en cuenta que para el transporte de información de voz sobre redes, es necesario ensamblar paquetes, la capacidad de canal requerida dependerá de la sobrecarga que generen estos paquetes. Para el envío de voz sobre redes LAN PLC se utiliza el protocolo RTP (Real-Time Transport Protocol / Protocolo de transporte en tiemporeal). Este protocolo a su vez se encapsula sobre el protocolo de transporte UDP (User Datagram Protocol / Protocolo de Datagrama de usuario), el que a su vez se encapsula sobre el protocolo de Internet (IP) y viaja sobre la red PLC. En la Fig. 1 se presenta de manera gráfica todo el proceso de encapsulamiento de la voz para ser transmitida en una red PLC.

Fig. 1. TRANSPORTE DE VOZ EN REDES PLC

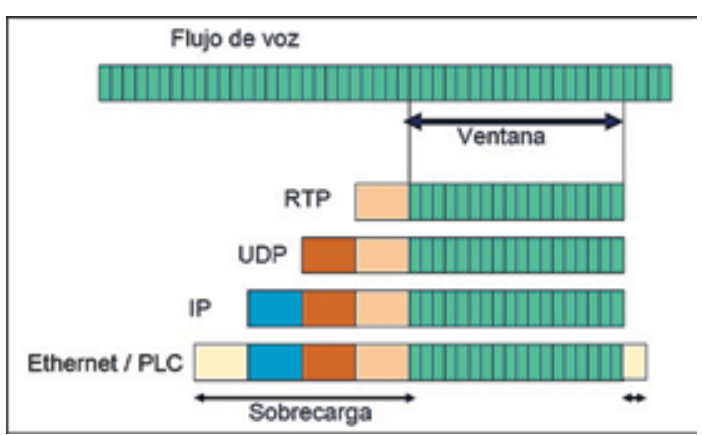

Fuente: autores. 
A continuación se presenta un ejemplo para el codec G.711. Para una ventana de $20 \mathrm{~ms}$ y codificación de audio G.711, se obtienen 160 bytes de voz por trama como se puede evidenciar en la Fig. 2.

Fig. 2. TRAMA PARA CODEC G.711

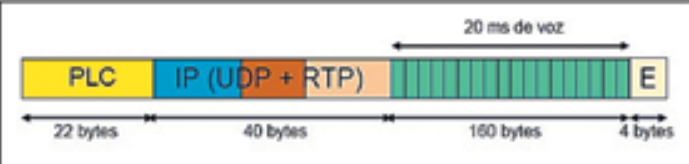

Fuente: autores.

El número de Bytes de voz/trama puede ser calculado de la siguiente forma:

Bytes de voz/trama $=64 \mathrm{kbps} * 20 \mathrm{~ms} / 8$ = 160 bytes

El encapsulamiento IP (incluyendo los protocolos RTP y UDP) agrega 40 bytes adicionales
Bytes de paquete $\mathrm{IP}=160+40=200$ bytes

La trama PLC adiciona otros 26 bytes:

Bytes de trama PLC $=200+26=226$ bytes

Para el ejemplo presentado se observa que cada $20 \mathrm{~ms}$ se genera 226 bytes que se deben transportar por la red LAN PLC. Ello corresponde a un ancho de banda de 90,4 Kbps, el cual es bastante mayor que el flujo de audio (64 kbps).

Capacidad de canal requerido $=226 * 8 /$ $20 \mathrm{~ms}=90.4 \mathrm{kbps}$

En la Tabla III se establece la capacidad de canal requerida para cada uno de los codecs más representativos en el manejo de VolP, en redes LAN sobre PLC (G.711, G.723 y G.729). Un aspecto importante por destacar es que la capacidad de canal requerida puede variar considerablemente dependiendo de codec usado y de la ventana seleccionada.

TABLA III

CAPACIDAD DE CANAL ASOCIADO A LOS CODECS PARA VOIP SOBRE PLC

\begin{tabular}{|c|c|c|c|c|c|}
\hline Tipo de Codec & $\begin{array}{c}\text { Duración de trama } \\
\text { (ms) }\end{array}$ & $\begin{array}{c}\text { Bytes de voz/ } \\
\text { trama }\end{array}$ & $\begin{array}{c}\text { Bytes de paque- } \\
\text { te IP }\end{array}$ & $\begin{array}{c}\text { Bytes de trama } \\
\text { PLC }\end{array}$ & $\begin{array}{c}\text { Ancho de Banda LAN } \\
\text { PLC (kbps) }\end{array}$ \\
\hline \multirow{3}{*}{ G.711 (64 kbps) } & 10 & 80 & 120 & 146 & 116.8 \\
\hline & 20 & 160 & 200 & 226 & 90.4 \\
\hline & 30 & 240 & 280 & 306 & 81.6 \\
\hline G.723.1 (6.3 kbps) & 30 & 24 & 64 & 90 & 23.9 \\
\hline G.723.1 (5.3 kbps) & 30 & 20 & 60 & 86 & 22.9 \\
\hline \multirow{3}{*}{ G.729 (8 kbps) } & 10 & 10 & 50 & 76 & 60.8 \\
\hline & 20 & 20 & 60 & 86 & 30.4 \\
\hline & 30 & 30 & 70 & 96 & 25.6 \\
\hline
\end{tabular}

\subsection{Planeación del experimento}

El rendimiento es sin duda uno de los aspectos de mayor interés dentro del análisis global en las redes LAN debido al efecto que este produce sobre el usuario final. El rendimiento puede ser definido según diversos puntos de vista, permitiendo con ello incorporar otras formas de evaluación dependiendo del objeto de interés en particular. Entre los parámetros más comunes para evaluar el rendimiento de una red se pueden mencionar: throughput, utilización del canal, latencia, jitter y porcentaje de paquetes perdidos. Sin embargo, en el presente artículo solo se considerarán throughput, latencia y jitter como parámetros para evaluar el rendimiento de la red para la transmisión de
VolP. A continuación se presentan las definiciones para cada uno de los parámetros mencionados:

- Throughput. Se define como la cantidad de información útil por unidad de tiempo que es transmitida en un canal de comunicaciones. Su unidad de medida es bits/seg.

- Jitter. Según la norma RFC 3393 se define como el valor estadístico relacionado con la variación del retardo entre paquetes; normalmente implementado como la variación de la demora en los paquetes consecutivos [20].

- Latencia. Se define como el tiempo que tarda un paquete en ser transmitido desde el host emisor hasta su llegada en el receptor, y se encuentra constituida principalmente por el 
tiempo de propagación y el tiempo de transmisión.

A continuación se describen las características de los elementos hardware y software utilizados para el desarrollo del experimento propuesto:

\section{Hardware}

- PCs: Los equipos de cómputo utilizados corresponden a computadores portátiles marca DELL con tarjeta de red marca Broadcom tipo Ethernet 1 Gbps y sistema operativo Windows 7.

- Cableado: El cableado eléctrico ubicado en las instalaciones donde se realizaron los experimentos, está instalado con alambre AWG (American Wire Gauge / Calibre de cable americano) número 12 con alimentación a 110 voltios y operando en frecuencia de $60 \mathrm{~Hz}$. Con referencia a los cables UTP, se utilizaron patch cords certificados de acuerdo con la norma ANSI/TIA/EIA-568-A y operando en Categoría 5e.

- Adaptadores PLC: Para implementar la red PLC se utilizaron adaptadores de red Power Line marca TP-LINK modelo PA-211. En la Fig. 3 y en la Tabla IV se presentan los adaptadores PLC usados y sus especificaciones técnicas.

Fig. 3. ADAPTADOR DE RED HOMEPLUG AV MARCA TP-LINK MODELO TL-PA211

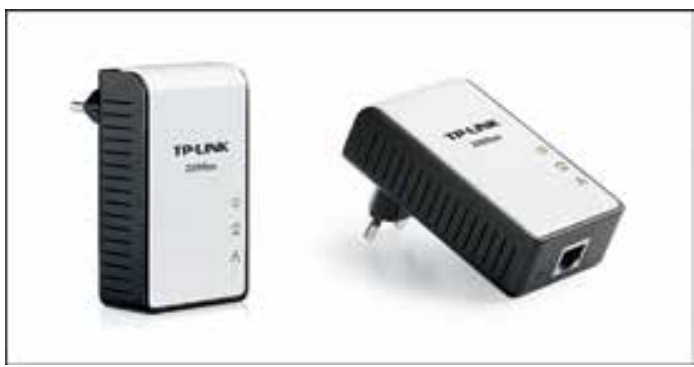

Fuente: http://www.tp-link.com/resources/images/products/gallery/TLPA211-01.jpg

TABLA IV

ESPECIFICACIONES TÉCNICAS DEL MODEM TP-LINK MODELO TL-PA211

\begin{tabular}{|l|l|}
\hline Característica & Detalle \\
\hline Estándares y protocolos & HomePlug AV, IEEE802.3, IEEE802.3u \\
\hline Interfaces & Puerto Ethernet a 10/100/1000 Mbps \\
\hline Tipo de enchufe & Unión Europea, Estados Unidos, Reino Unido, Australia \\
\hline Botones & Botón de emparejamiento \\
\hline Peso & 103 Gramos \\
\hline Indicador LED & PWR, PLC, ETH \\
\hline Dimensiones (W X D X H) & $3,6 \times 2,2 \times 1,2$ pulgadas $(93 \times 56 \times 30$ mm) \\
\hline Consumo de potencia & $<3$ W \\
\hline Alcance & 300 metros dentro de la casa/oficina \\
\hline Tecnología de modulación & OFDM \\
\hline Funciones avanzadas & $\begin{array}{l}\text { Función QoS (calidad del servicio), selección inteligente } \\
\text { de canal }\end{array}$ \\
\hline Encriptación & Encriptación AES de 128 bits \\
\hline
\end{tabular}

Fuente: http://www.tp-link.com/co/products/details/?categoryid=\&model=TL-PA211\#spec

\section{Software}

En la actualidad existen diferentes herramientas software que permiten realizar mediciones sobre una red. La mayoría de herramientas operan mediante configuraciones cliente/servidor, enviando paquetes de un host a otro, generando situaciones de tráfico controladas y aleatorias, permitiendo variar el tipo de protocolo de transmisión, TCP o UDP, el tamaño del paquete, y en algunas ocasiones la tasa de transferencia. En vista de lo anterior y con el fin de facilitar los procesos de generación y captura de tráfico relacionado con voz sobre IP, se utilizó el aplicativo software denominado Generador de Tráfico Distribuido de Internet v2.61 (D-ITG / Distributed Internet Traffic Generator), desarrollado en el Departamento de Informática y Sistémica de la Universidad "Federico II" de Italia [21]. En la Fig. 4 se presenta la interfaz gráfica de usuario correspondiente al generador de tráfico D-ITG. 
Fig. 4. GUI DEL GENERADOR DE TRÁFICO D-ITG

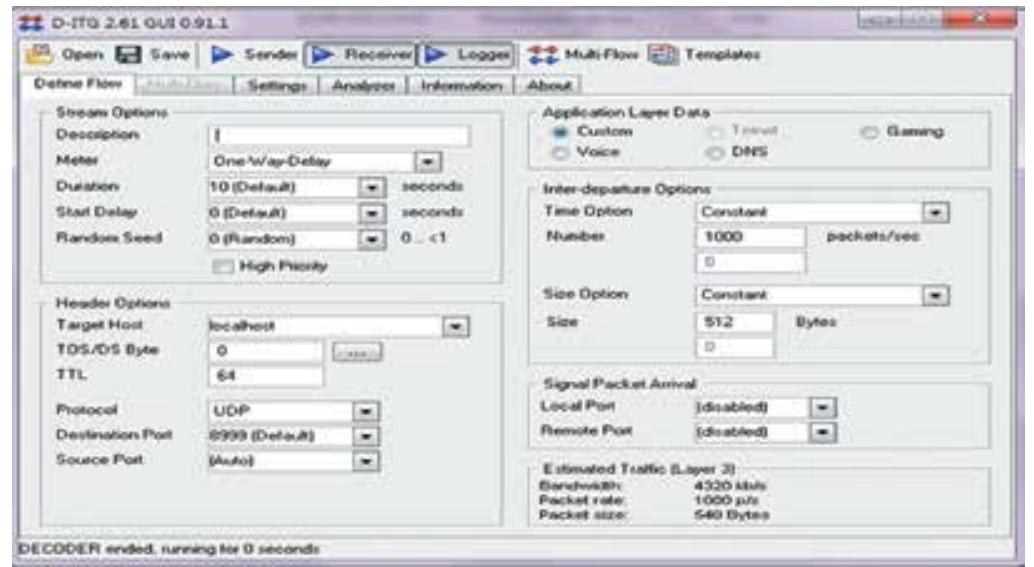

Fuente: autores

D-ITG es una herramienta de libre distribución, amplia aceptación y muy utilizada para generación y análisis de tráfico en todo tipo de redes de datos, operando sobre diversos medios de transmisión [22]. Además incorpora las principales características que debe poseer un generador de tráfico, como son:

- Adaptabilidad a redes heterogéneas multidimensionales.

- Manejo de tráfico multimedia (video, voz, entre otros).

- Incorporación de los principales codecs de audio (G711, G723 y G729).

- Soporte de múltiples protocolos (IPv4, IPv6, ICMP, TCP, UDP, SCTP, etc.).

- Control distribuido remoto tanto para emisores como para receptores de tráfico.

- Generación de múltiples flujos de tráfico hacia diferentes receptores en la red.
- Configuración de cantidad y tamaño de paquetes emitidos, además de sus correspondientes tiempos de emisión.

- Medición de múltiples métricas de red, asociadas con el rendimiento, como throughput, jitter, latencia, porcentaje de paquetes perdidos, entre otros.

\subsection{Descripción del experimento}

Para el desarrollo del experimento se dispone la configuración de una red LAN operando sobre el cableado eléctrico y haciendo uso de adaptadores HomePlug AV marca TP-LINK, modelo TL-PA211 en cuyos extremos se conectan los equipos de usuario final (PCs) con cables ethernet. Los equipos utilizados se configuran con direcciones de red tipo IP clase $C$ y su esquema de conexión se aprecia en la Fig. 5.

Fig. 5. CONFIGURACIÓN DE RED HOMEPLUG AV USADA EN EL EXPERIMENTO

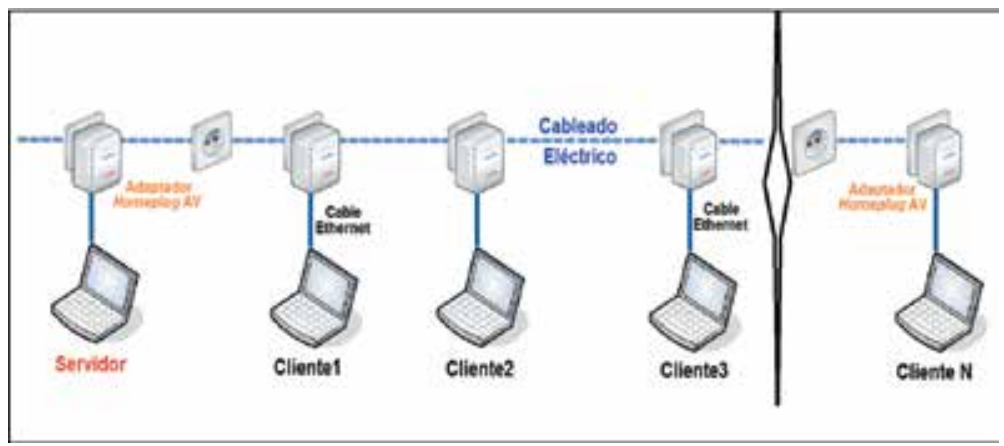


En el esquema propuesto uno de los PC, mediante el software D-ITG se configura como servidor y los equipos restantes se configuran como clientes. Los clientes tienen la función de generar tráfico tipo VoIP, bajo parámetros de configuración específicos acordes con el objetivo del experimen- to, en el servidor se realiza la captura y el análisis de los paquetes que circulan por la red HomePlug AV. Los parámetros de configuración específicos en el D-ITG para el desarrollo del experimento se presentan en la Tabla V.

TABLA V

PARÁMETROS PARA EL DESARROLLO DEL EXPERIMENTO

\begin{tabular}{|l|l|}
\hline Característica & Detalle \\
\hline Tiempo de duración $x$ toma de datos & 30 segundos \\
\hline Cantidad de paquetes $x$ toma de datos & 1000 paquetes \\
\hline Direccionamiento usado en las estaciones & IP clase C \\
\hline Tipos de Codec utilizados para la generación de tráfico de voz & G.711, G.723 y G.729 \\
\hline Tamaño de paquete & $\begin{array}{l}\text { Depende del tipo de Codec utilizado. En la Tabla X se ilustra el tamaño del paquete } \\
\text { en función del tipo de Codec establecido. }\end{array}$ \\
\hline Cantidad de estaciones que forman parte de la red PLC. & $1,2,5$ y 10 estaciones \\
\hline
\end{tabular}

Fuente: autores.

Con base en el objetivo propuesto se seleccionó un diseño experimental de tipo factorial mixto, el cual busca establecer el grado de influencia que cada uno de los factores ejerce sobre la variable de salida [23]. Para el caso particular, los factores o variables de entrada serán el número de estaciones activas y el tipo de codec. Por otro lado, las variables de salida son: throughput, jitter y latencia. Los valores que serán considerados por cada factor se encuentran claramente establecidos en la Tabla V.

Antes de dar inicio a las campañas de muestreo, es necesario determinar el número de réplicas que deben ser consideradas como mínimo para cada condición específica del experimento, con el fin de garantizar que la cantidad de mues- tras que serán tomadas durante el desarrollo del experimento es la adecuada, [24] menciona que para realizar un estudio de carácter empírico o experimental, las variables que intervienen en la determinación del tamaño de la muestra son: el nivel de confianza, la potencia de la prueba y la magnitud de la diferencia, para las cuales se han establecido valores de 0.05, 0.7 y magnitud de diferencia grande, respectivamente. De acuerdo con los valores registrados en la Tabla VI, se consideró un tamaño de muestra igual a cinco (5), el cual corresponde al valor establecido para una relación de $3 \times 4$, teniendo en cuenta que el factor "codec" comprende 3 niveles (Codec G.711, G.723 y G.729) y el factor "Número de estaciones" comprende 4 niveles (1, 2, 5 y 10 PCs).

TABLA VI

TAMAÑO DE MUESTRA PARA DISEÑO FACTORIAL CON DOS (2) FACTORES

\begin{tabular}{|c|c|c|c|c|}
\hline \multirow{2}{*}{ Niveles Factor1 x Niveles Factor2 } & \multirow{2}{*}{$\begin{array}{c}\text { Potencia } \\
(\mathbf{1} \boldsymbol{\beta})\end{array}$} & \multicolumn{3}{|c|}{ Magnitud de la diferencia } \\
\cline { 2 - 5 } & 0,70 & 152 & 25 & 11 \\
\hline \multirow{2}{*}{$2 \times 2$} & 0,80 & 193 & 32 & 13 \\
\cline { 2 - 5 } & 0,70 & 127 & 21 & 9 \\
\hline \multirow{2}{*}{$2 \times 3$} & 0,80 & 158 & 26 & 11 \\
\cline { 2 - 5 } & 0,70 & 109 & 18 & 8 \\
\cline { 2 - 5 } & 0,80 & 134 & 22 & 9 \\
\hline \multirow{2}{*}{$3 \times 4$} & 0,70 & 85 & 14 & 6 \\
\cline { 2 - 5 } & 0,80 & 106 & 18 & 7 \\
\hline \multirow{2}{*}{$3 \times 4$} & 0,70 & 73 & 12 & 5 \\
\cline { 2 - 5 } & 0,80 & 90 & 15 & 6 \\
\hline \multirow{2}{*}{$4 \times 4$} & 0,70 & 55 & 9 & 4 \\
\cline { 2 - 5 } & 0,80 & 67 & 12 & 5 \\
\hline
\end{tabular}

Fuente: [24]. 
En vista de lo anterior, el diseño factorial mixto corresponde a $3 \times 4=12$ puntos experimentales y $3 \times 4 \times 5=60$ mediciones por variable de respuesta.

\subsection{Resultados del experimento}

En la Tabla VII se presenta el consolidado referente a la matriz de diseño para las tres variables objeto de estudio, donde se registran los valores obtenidos experimentalmente.

TABLA VII

MATRIZ DE DISEÑO PARA THROUGHPUT, LATENCIA Y JITTER

\begin{tabular}{|c|c|c|c|c|c|c|c|c|c|}
\hline \multirow{2}{*}{$\begin{array}{c}\text { No. de estaciones } \\
\text { o teléfonos }\end{array}$} & \multicolumn{3}{|c|}{ Throughput [kbps] } & \multicolumn{3}{|c|}{ Latencia [ms] } & \multicolumn{3}{|c|}{ Jitter [ms] } \\
\hline & G711 & G723 & G729 & G711 & G723 & G729 & G711 & G723 & G729 \\
\hline \multirow{5}{*}{1} & 76,107 & 9,519 & 13,162 & 0,756 & 0,78 & 0,78 & 0,27 & 0,745 & 0,374 \\
\hline & 77,36 & 9,567 & 13,027 & 0,762 & 0,857 & 0,619 & 0,302 & 0,71 & 0,227 \\
\hline & 76,593 & 9,484 & 13,902 & 0,79 & 0,735 & 0,717 & 0,306 & 0,579 & 0,383 \\
\hline & 76,392 & 8,885 & 13,135 & 0,715 & 0,872 & 0,792 & 0,266 & 0,781 & 0,276 \\
\hline & 76,149 & 8,792 & 13,043 & 2,81 & 0,709 & 0,616 & 0,515 & 0,575 & 0,38 \\
\hline \multirow{5}{*}{2} & 150,866 & 17,911 & 25,888 & 1,326 & 1,451 & 1,803 & 0,302 & 0,777 & 0,395 \\
\hline & 151,078 & 18,613 & 26,891 & 0,761 & 3,72 & 1,689 & 0,297 & 0,752 & 0,492 \\
\hline & 150,623 & 19,027 & 27,53 & 1,84 & 1,979 & 0,902 & 0,344 & 0,785 & 0,386 \\
\hline & 153,057 & 18,526 & 27,607 & 6,374 & 1,783 & 0,178 & 0,697 & 0,703 & 0,478 \\
\hline & 153,418 & 17,989 & 27,422 & 1,668 & 2,984 & 2,751 & 0,401 & 0,84 & 0,371 \\
\hline \multirow{5}{*}{5} & 376,387 & 44,88 & 66,04 & 2,184 & 2,25 & 3,352 & 0,41 & 0,905 & 0,586 \\
\hline & 385,237 & 45,04 & 68,031 & 5,428 & 4,826 & 4,766 & 0,591 & 1,038 & 0,688 \\
\hline & 385,379 & 45,255 & 65,593 & 4,455 & 3,303 & 1,761 & 0,561 & 0,973 & 0,525 \\
\hline & 391,367 & 44,757 & 65,389 & 3,872 & 2,674 & 4,738 & 0,582 & 1,006 & 0,581 \\
\hline & 368,269 & 43,454 & 67,438 & 4,173 & 4,245 & 1,848 & 0,459 & 0,938 & 0,618 \\
\hline \multirow{5}{*}{10} & 796,379 & 92,463 & 137,481 & 6,295 & 8,039 & 7,727 & 1,394 & 1,474 & 1,718 \\
\hline & 737,871 & 90,308 & 137,915 & 7,475 & 8,214 & 6,977 & 1,67 & 1,673 & 1,526 \\
\hline & 793,945 & 91,772 & 133,984 & 6,934 & 8,14 & 6,465 & 1,294 & 1,753 & 1,205 \\
\hline & 729,304 & 88,573 & 134,157 & 6,482 & 8,045 & 7,492 & 1,384 & 1,595 & 1,762 \\
\hline & 751,323 & 89,437 & 128,305 & 7,321 & 8,217 & 6,621 & 1,521 & 1,672 & 1,204 \\
\hline
\end{tabular}

Fuente: autores.

\subsection{Análisis de resultados}

En primera instancia se procedió a realizar un análisis de varianza (ANOVA) para cada una de las variables objeto de estudio, teniendo en cuenta que esta es la técnica de análisis sugerida en diseños experimentales de tipo factorial [25]. En un diseño factorial de dos (2) factores la variable de respuesta () puede describirse mediante el modelo de efectos dado por (1) [26]:

$$
\begin{aligned}
& Y_{i j l}=\mu+A_{i}+B_{j}+(A B)_{i j}+\varepsilon_{i j l} \\
& i=1,2, . . a ; j=1,2, \ldots b ; l=1,2 \ldots n
\end{aligned}
$$

donde,

$a=$ Codec utilizado.

$b=$ número de estaciones en la red HomePlug AV.

$n=$ número de réplicas del experimento.

$Y_{i j l}=$ Variable de salida (throughput, latencia o jitter), para un codec $(i)$, con un número de estaciones en la red HomePlug AV $(b)$ y replicación $(l)$

$\mu=$ Media general de la variable de salida, independiente de cualquiera de los factores considerados en el experimento. 
$A_{i}=$ Efecto del Codec usado $(i)$

$B_{j}=$ Efecto del número de estaciones $(j)$.

$(A B)_{i j}=$ Efecto de la interacción entre los factores Codec y número de estaciones.

$\varepsilon_{i j k l}=$ Error aleatorio.

Teniendo en cuenta que el experimento realizado presenta tres (3) posibles efectos de interés ( $A$, B y $A B$ ) es necesario plantear tres (3) hipótesis nulas $\left(H_{0}\right)$ y cada una emparejada con una hipótesis alternativa $\left(H_{A}\right)$, asociadas con cada uno de los efectos posibles, con el fin de contrastar su validez [23]. En vista de lo anterior, las hipótesis propuestas para los dos (2) factores y sus interacciones son las siguientes:

$$
\begin{aligned}
& H_{0}: \text { Efecto } A=0, H_{A}: \text { Efecto } A \neq 0 \\
& H_{0}: \text { Efecto } B=0, H_{A}: \text { Efecto } B \neq 0 \\
& H_{0}: \text { Efecto } A B=0, H_{A}: \text { Efecto } A B \neq 0
\end{aligned}
$$

En las Tablas VII, VIII y IX se presentan los resultados correspondientes al ANOVA para las variables de throughput, latencia y jitter, respectivamente.

TABLA VIII

ANOVA COMPLETO PARA THROUGHPUT

\begin{tabular}{|l|r|r|r|r|r|}
\hline \multicolumn{1}{|c|}{ Fuente de variación } & $\begin{array}{r}\text { Suma de cuadrados } \\
\text { (SC) }\end{array}$ & Gr. Lib & $\begin{array}{c}\text { Cuadrado medio } \\
\text { (CM) }\end{array}$ & $\begin{array}{c}\text { Cociente-F } \\
\text { (Fo) }\end{array}$ & $\mathrm{p}$-Valor \\
\hline A: Codec & 1143418,91 & 2 & 571709,45 & 6259,75 & $<0,0001$ \\
\hline B: No. de estaciones & 794482,37 & 3 & 264827,46 & 2899,64 & $<0,0001$ \\
\hline Interacción AxB & 691634,18 & 6 & 115272,36 & 1262,14 & $<0,0001$ \\
\hline Error & 4383,89 & 48 & 91,33 & & \\
\hline TOTAL & 2633919,34 & 59 & & & \\
\hline
\end{tabular}

Fuente: autores.

De acuerdo con [27] un efecto se puede considerar estadísticamente significativo cuando el $p-$ Valor es menor al valor establecido para Por lo tanto, se tomarán como estadísticamente significativos los valores cuyo $p-$ Valor $<0,05$. En la Tabla VIII se observa que los tres efectos propuestos presentan un $p-$ Valor inferior a 0,05
(A, B y AB), por lo cual se podría manifestar tanto el tipo de codec como el número de estaciones, y la interacción conjunta de estos dos factores presentan un efecto estadísticamente significativo sobre el throughput en un 95\% de confianza, aspecto por el que en los tres casos la hipótesis $H_{0}$ se rechaza.

\begin{tabular}{|c|c|c|c|c|c|}
\hline Fuente de variación & $\begin{array}{l}\text { Suma de cuadrados } \\
\text { (SC) }\end{array}$ & Gr. Lib & $\begin{array}{l}\text { Cuadrado medio } \\
\text { (CM) }\end{array}$ & $\begin{array}{c}\text { Cociente-F } \\
\text { (Fo) }\end{array}$ & $\mathrm{p}$-Valor \\
\hline A: Codec & 3,74 & 2 & 1,87 & 1,72 & 0,19 \\
\hline B: Nro. de estaciones & 356,55 & 3 & 118,85 & 108,91 & $<.0001$ \\
\hline Interacción AxB & 5,65 & 6 & 0,94 & 0,86 & 0,528 \\
\hline Error & 52,38 & 48 & 1,09 & & \\
\hline TOTAL & 418,32 & 59 & & & \\
\hline
\end{tabular}

TABLA IX

ANOVA COMPLETO PARA LA LATENCIA

Fuente: autores.

Acorde con el resultado obtenido en la Tabla IX, se puede observar que solo el efecto $B$ presenta un $p$-Valor inferior a 0.05 , por lo cual se podría manifestar que para este caso solo el número de estaciones activas en la red PLC presenta un efecto estadísticamente significativo sobre la latencia que puede llegar a presentar un proceso de transmisión de VolP, con un 95 \% de confianza, rechazándose la hipótesis $H_{o}$ para este factor. 
TABLA X

ANOVA COMPLETO PARA JITTER

\begin{tabular}{|c|c|c|c|c|c|}
\hline Fuente de variación & $\begin{array}{l}\text { Suma de cuadrados } \\
\text { (SC) }\end{array}$ & Gr. Lib & $\begin{array}{l}\text { Cuadrado medio } \\
\text { (CM) }\end{array}$ & $\begin{array}{l}\text { Cociente-F } \\
\text { (Fo) }\end{array}$ & $\mathrm{p}$-Valor \\
\hline A: Codec & 1,38 & 2 & 0,69 & 46,80 & $<0,0001$ \\
\hline B: Nro. de estaciones & 10,94 & 3 & 3,65 & 247,93 & $<0,0001$ \\
\hline Interacción AxB & 0,12 & 6 & 0,02 & 1,40 & 0,23 \\
\hline Error & 0,71 & 48 & 0,01 & & \\
\hline TOTAL & 13,14 & 59 & & & \\
\hline
\end{tabular}

Fuente: autores.

En la Tabla X se puede observar que los efectos A y B presentan un inferior a 0.05, por lo cual se podría manifestar que para este caso, tanto el número de estaciones como el tipo de codec muestran un efecto estadísticamente significativo sobre el jitter que puede llegar a presentar un proceso de transmisión de VolP, con un $95 \%$ de confianza, rechazándose la hipótesis para estos dos factores.

\subsection{Comparaciones múltiples con el mejor tratamiento}

Con el fin de comparar el resultado obtenido por los tres tipos de codec e identificar aquellos que arrojan el mejor resultado de acuerdo con el problema propuesto, se hará uso de la prueba de Dunnett, con un nivel de confianza específico. Como el interés particular radica en determinar cuál es el tratamiento que mejor minimiza los niveles de latencia y jitter, el proceso es el siguiente:

Paso 1: Se calcula la diferencia $D_{i}$ entre cada una de las medias $\bar{y}_{l}$ (una por tratamiento), y la menor media de los restantes $\min _{j \neq i} \bar{y}_{j}$, como se presenta a continuación (2):

$$
D_{i}=\bar{y}_{l}-\min _{j \neq i} \bar{y}_{j} \forall i=1,2, \ldots, t
$$

Donde $\min _{j \neq i} \mu_{j}$ es la media mínima de los tratamientos sin incluir a $\mu_{i}$.

Paso 2: Se procede a calcular el estadístico de Dunnet $(M)$

$$
M=d_{\alpha, k, v} \sqrt{\frac{2 S^{2}}{r}}
$$

Donde $d_{\alpha, k, v}$ obedece al estadístico tabulado correspondiente a las comparaciones múltiples con el mejor bajo la prueba de Dunnett [28]. $S^{2}$ y $r$ corresponden a la varianza expermiental (Cuadrado medio del error $\mathrm{CME}$ ) y el número de repeticiones, respectivamente.
Paso 3: Para calcular los intervalos de confianza simultáneos (ICS) con un 100(1- $\alpha$ )\% de nivel de confianza, se deben tener en cuenta las siguientes expresiones:

Límite Inferior

$$
L=\left\{\begin{array}{c}
D_{i}-M \text { Si } D_{i}-M<0 \\
0 \text { de otra forma }
\end{array}\right.
$$

Límite Superior

$$
U=\left\{\begin{array}{c}
D_{i}-M \text { Si } D_{i}+M>0 \\
0 \text { de otra forma }
\end{array}\right.
$$

Paso 4: Aquellos tratamientos en los cuales se incluya el cero entre los límites superior e inferior, serán considerados como el mejor o los mejores tratamientos. Si solo interesa determinar el mejor tratamiento sin importar cuánto difieren sus efectos entre sí y considerando que lo que se desea es identificar el tratamiento que mejor minimice los niveles de latencia, basta con seleccionar aquel tratamiento que presente la menor media [28].

Paso 5: Con base en los valores registrados en la Tabla VII para el máximo número de estaciones conectadas en la red PLC (10 estaciones), se procede a calcular los valores correspondientes a la suma de cuadrados del error $(S C E)$, los grados de libertad (G.L.), el error cuadrático medio $(C M E)$ y $M$; para cada uno de los codec utilizados, teniendo en cuenta el número de tratamientos $\left(N_{t}=3\right)$ y el número de muestras o réplicas $\left(N_{m}=5\right)$ por tratamiento. El resultado de este proceso es el siguiente:

$$
\begin{aligned}
& S C E=\sum_{i=1}^{N_{t}} \sum_{j=1}^{N_{m}}\left[y_{i j}-\mu_{i}\right]^{2}=0,873+0,023+0,995 \\
& S C E=1,89 \\
& G . L=N_{t}\left(N_{m}-1\right)=3(5-1)=12 \\
& C M E=\frac{S C E}{N_{t}\left(N_{m}-1\right)}=0,157
\end{aligned}
$$


El valor del estadístico de Dunnett para $\alpha=0,05, k=t-1=2 \quad$ y $\quad v=G . L \quad$ es: $d_{\alpha, k, v}=2,18$, el cual puede ser consultado en [25]. Reemplazando los valores, el valor de $\mathrm{M}$ está dado por:

$$
M=d_{\alpha, k, v} \sqrt{\frac{2 S^{2}}{r}}=2,18 \sqrt{\frac{2 C M E}{N_{m}}}=0,54
$$

En la Tabla XI se presenta el resumen de cada uno de los parámetros requeridos para realizar la prueba de Dunnett.

TABLA XI

PRUEBA DE DUNNET PARA LA LATENCIA

\begin{tabular}{|c|c|c|c|c|c|c|}
\hline Codec & Media $\left(\mu_{i}\right)$ & Min $\left(\mu_{i \neq j}\right)$ & $\mathrm{M}$ & $D_{i}$ & $L=D_{i}-M$ & $U=D_{i}+M$ \\
\hline G 711 & 6,9 & 7,05 & 0,54 & $-0,15$ & $-0,69$ & 0,39 \\
\hline G 723 & 8,13 & 6,9 & 0,54 & 1,23 & 0,69 & 1,77 \\
\hline G 729 & 7,05 & 6,9 & 0,54 & 0,15 & $-0,39$ & 0,69 \\
\hline
\end{tabular}

Fuente: autores.

Acorde con los resultados registrados en la Tabla XI, se podría manifestar que al realizar la prueba de Dunnett solo los codec G711 y G729 podrían ser considerados como los mejores, debido a que los intervalos de confianza simultáneos incluyen el cero entre los límites superior e inferior. Sin embargo, al evaluar la diferencia $D_{i}$ se observa que codec G711 es el que presenta la menor media y adicionalmente es el único de los tres codec que cumple con la condición $\mu_{i}-\max _{j \neq i} \mu_{j} \leq 0$, lo cual indica que codec G711 puede considerarse como el mejor de los tres codec utilizados. Adicionalmente, al observar los resultados del experimento en la Tabla VII, el codec G711 es el que mayores valores de throughput registró, aspecto por el cual se podría llegar a inferir que el codec G711 es el que mejor rendimiento ofrece a la hora de realizar procesos de transmisión de VolP sobre una red PLC en un contexto residencial.

\section{CONCLUSIONES}

Ante la necesidad de ofrecer adecuados niveles de QoS en aplicaciones de VolP en redes PLC, HPAV hace uso de un mecanismo de acceso al medio híbrido, incorporando TDMA con el fin de cumplir con el objetivo propuesto, el cual divide el periodo de tiempo reservado $(16,66 \mathrm{~ms})$ en $N$ slots de tiempo iguales, destinando a cada estación el tiempo de un slot. En virtud de lo anterior, se propuso un diseño experimental de tipo factorial mixto, con el fin de evaluar el rendimiento de la red PLC para la transmisión de VoIP, el cual permitió analizar los parámetros de throughput, latencia y jitter; en función de dos factores: tipo de codec y el número de estaciones activas que forman parte de la red. Con base en los resultados obtenidos mediante un análisis de varianza para cada uno de los parámetros de rendimiento seleccionados, se logró identificar cuales factores influyen de manera significativa sobre el throughput, la latencia y el jitter, lo cual puede ser considerado como un aspecto de gran importancia a la hora de optimizar el rendimiento de una red PLC. Adicionalmente, se pudo evidenciar que el uso de la tecnología PLC puede ofrecer adecuados niveles de QoS durante el proceso de transmisión de VolP, teniendo en cuenta que los valores de latencia y de jitter se mantuvieron por debajo de los $150 \mathrm{~ms}$, acorde con las recomendaciones establecidas en [29].

Al evaluar los valores de latencia generados por los codec G711, G723 y G729 en el interior de la red PLC mediante la prueba de Dunnett, con el fin de identificar cuál o cuáles de ellos podrían ser considerados como la mejor alternativa a la hora de transmitir VolP, se pudo establecer que, de acuerdo con los resultados obtenidos el codec G711 y el G729 arrojaron los mejores resultados, minimizando los niveles de latencia presentes en la red PLC. Sin embargo, el codec G711 fue el que presentó la menor media y adicionalmente fue el único de los tres codec que cumplió con la condición $\mu_{i}-\max _{j \neq i} \mu_{j} \leq 0$, aspecto por el cual el codec $\mathrm{G} 711$ puede considerarse como el mejor de los tres codec utilizados. Adicionalmente, el codec G711 fue el que mayores valores de throughput registró durante el experimento, reflejando con ello, que el codec G711 es el que mejor rendimiento podría ofrecer a la hora de realizar procesos de transmisión de VoIP en una red PLC y bajo un contexto residencial. Es importante mencionar 
que aunque el análisis realizado está restringido a 10 estaciones, se sugiere en futuros estudios evaluar el rendimiento de una red PLC para un número mayor de estaciones e incluso en condiciones de canal en estado de saturación.

\section{REFERENCIAS}

[1] J. Ekanayake, N. Jenkins, and K. Liyanage, Smart grid: technology and applications, 1a ed. West Sussex, Inglaterra: John Wiley \& Sons, 2012.

[2] C. Jin and T. Kunz, "Smart home networking: Combining wireless and powerline networking," in 2011 7th International Wireless Communications and Mobile Computing Conference, 2011, pp. 1276-1281.

[3] N. Anatory, J. \& Theethayi, Broadband Power-Line Communication Systems: Theory and Applications., 1a ed. Southampton, England: WIT Press, 2010.

[4] H. Latchman, K. Srinivas, L. Yonge, and S. Gavette, Homeplug AV and IEEE 1901: A Handbook for PLC Designers and Users, 1a ed. New Jersey, USA: Wiley-IEEE Press, 2013.

[5] L. D. Andreou G., Manitsas E., "Finite element characterization of LV power distribution lines for high frequency communications signals," Proc. 7th Int. Symp. Power-Line Commun. its Appl., pp. 109-119, 2003.

[6] G. S. Banwell T., "A new approach to the modelling of the transfer function of the power line channel," Proc. 5th Int. Symp. Power-Line Commun. its Appl., pp. 319324, 2009.

[7] M. Zimmermann and K. Dostert, "A multipath model for the powerline channel," IEEE Trans. Commun., vol. 50, no. 4, pp. 553-559, Apr. 2002.

[8] B. G., "System architecture for power-line communication and consequences for modulation and multiple access," 7 th Int. Symp. Power-Line Commun. its Appl., 2003.

[9] H. Hrasnica, A. Haidine, and R. Lehnert, Broadband Powerline Communications Networks Network Design, 1a ed. West Sussex, Inglaterra: John Wiley \& Sons, 2004.

[10] Ministerio de las Tecnologías de la Información y Comunicaciones de Colombia, "Plan Nacional de Tecnologías de la Información y las Comunicaciones 20082019," 2008.

[11] Ministerio de las Tecnologías de la Información y Comunicaciones de Colombia, "Plan Vive Digital Colombia. Documento Vivo del Plan,” 2011.

[12] Departamento Nacional de Planeación, "Vision Colombia II Centenario 2019. Resumen Ejecutivo," 2005.
[13] P. J. Piñero-Escuer, J. Malgosa-Sanahuja, and P. Manzanares-Lopez, "Homeplug-AV CSMA/CA Evaluation in a Real In-Building Scenario," IEEE Commun. Lett., no. June, pp. 683-685, 2011.

[14] FCC, "Federal Communications Commission." [Online]. Available: http://www.fcc.gov/.

[15] N. Wittenberg, Understanding voice over IP technology, 1a ed. New York, USA: Delmar Cengage Learning, 2009.

[16] UIT-T, "Recommendation G.711: Pulse Code Modulation (PCM) of voice frequencies," 1988.

[17] UIT-T, "Recommendation G.723.1: Dual rate speech coder for multimedia communications transmitting at 5.3 and $6.3 \mathrm{kbit} / \mathrm{s}, " 2006$.

[18] UIT-T, "Recommendation G.729: Coding of speech at 8 kbits using Conjugate- Structure Algebraic-Code-Excited Linear-Prediction (CS-ACELP)," 2007.

[19] A. Tanenbaum and D. Wheteral, Computer Networks, 5a ed. Boston, USA: Pearson-Prentice Hall, 2011.

[20] C. Demichelis and P. Chimento, "RFC 3393 IP packet delay variationmetric for IP performance metrics (IPPM)," Status Propos. Stand., p. 21, 2002.

[21] S. . P. A. Avallone, "D-ITG Distributed Internet Traffic Generator." [Online]. Available: http://traffic.comics. unina.it/software/ITG/.

[22] A. Botta, A. Dainotti, and A. Pescapé, "A tool for the generation of realistic network workload for emerging networking scenarios," Comput. Networks, vol. 56, no. 15, pp. 3531-3547, Oct. 2012.

[23] H. Gutiérrez, Análisis y diseño de experimentos, 1a ed. México D.F., México: McGraw-Hill, 2008.

[24] P. Morales Vallejo, "Tamaño necesario de la muestra: ¿Cuántos sujetos necesitamos ?,” p. 24, 2012.

[25] D. Montgomery, Diseño y Análisis de Experimentos, 2a ed. México D.F., México: Limusa Wiley, 2004.

[26] R. Martínez and N. Martínez, Diseño de Experimentos. Análisis de datos estándar y no estándar, 1 ed. Bogotá, D.C., 1997.

[27] W. Moreno, Aplicaciones al diseño y análisis de experimentos. Bucaramanga, 2002.

[28] R. Kuelh, Diseño de experimentos: principios estadísticos para el análisis y diseño de investigaciones, 2nd. ed. Thompson, 2001.

[29] Cisco, "Quality of service for voice over IP," San Francisco, 2001. 